\title{
Accounting for Language Background
}

\section{Differences in the Australian National}

Assessments - Literacy and Numeracy

-

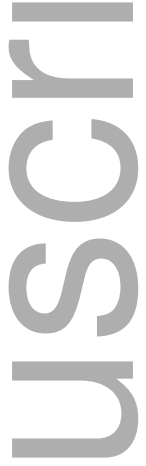

Gary N Marks ${ }^{1 *}$

Bruce Phillips ${ }^{2}$

1. Social and Political Sciences, University of Melbourne, g.marks@unimelb.edu.au

2.Policy Adviser, Catholic Education Melbourne, bphillips@cem.edu.au

*Corresponding author (Marks)

Acknowledgment: We would like to thank of Tony Alderton, Catholic Education Melbourne, for preparation of the data.

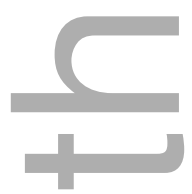

Gary N. Marks is an honorary principal fellow in the Department of Sociology, Social and Political Sciences at the University of Melbourne. He has publications in four main areas: educational outcomes (student achievement, university entrance performance, school completion and early school leaving, school effects), labour market outcomes (e.g. employment, unemployment, occupational attainment, occupational mobility, income and wealth) and social outcomes (e.g. wellbeing, leaving home and family formation). His work has a particular emphasis on changes over time This is the author manuscript accepted for publication and has undergone full peer review but has not been through the copyediting, typesetting, pagination and proofreading process, which may lead to differences between this version and the Version of Record. Please cite this article as doi: 10.1002/AJS4.108

This article is protected by copyright. All rights reserved 
and cross-national differences in social stratification, social inequality and the contribution of genetics to educational and socioeconomic outcomes.

Bruce Phillips is an education economist working in Catholic education in Victoria. He specialises in data analysis in the education sector, and in particular, assessments of school performance and school funding models.

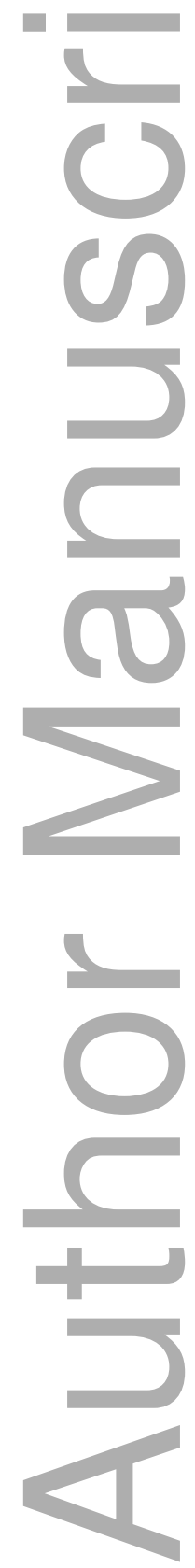

This article is protected by copyright. All rights reserved 


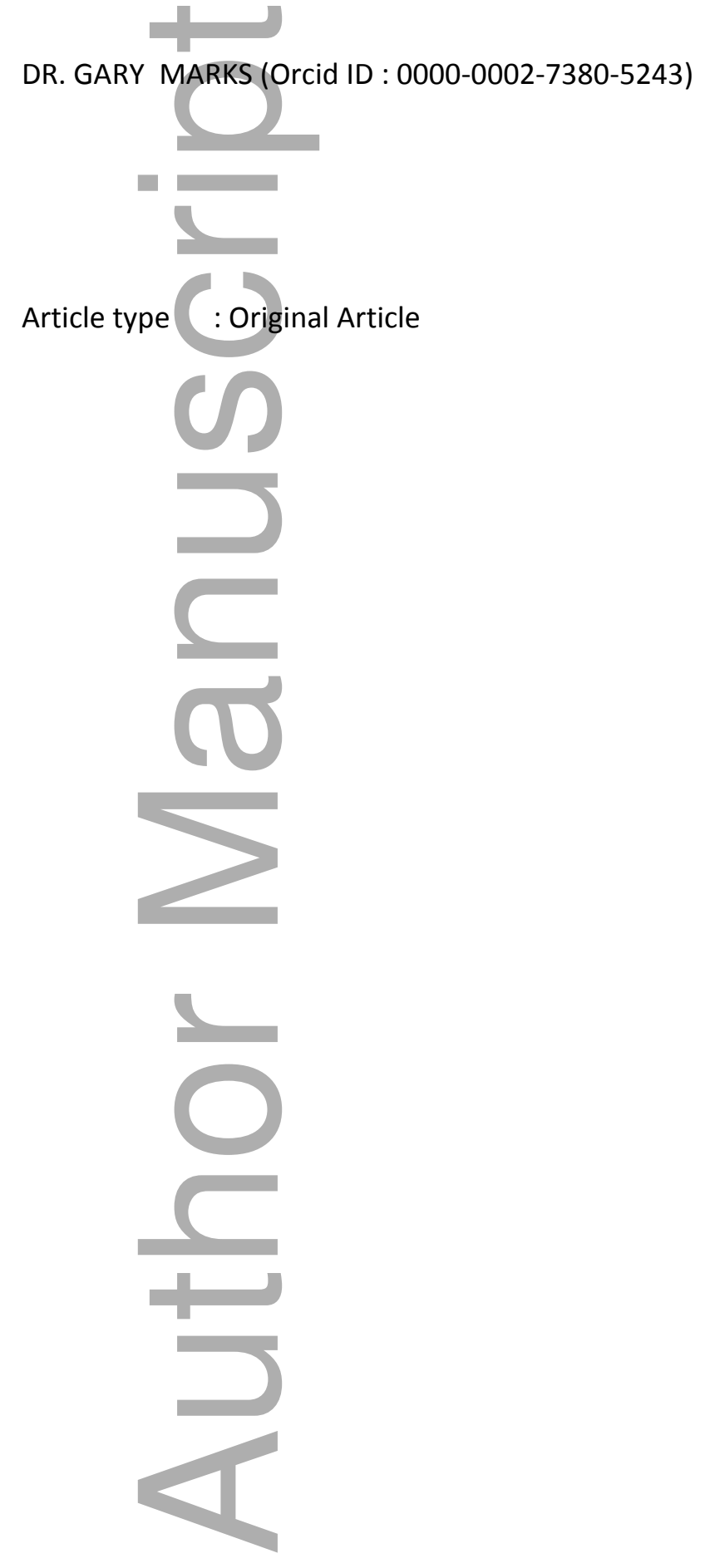

This article is protected by copyright. All rights reserved 


\title{
Accounting for Language Background Differences in the Australian National Assessments-Literacy and Numeracy
}

\begin{abstract}
This study examines language background differences in the Australian National Assessments - Literacy and Numeracy (NAPLAN), and the extent that language background differences are accounted for by socioeconomic background, schools and contemporary influences. There are sizable, sometimes very large, differences in performance by language background. Students with Eastern and Southern Asian language backgrounds exhibited high, sometimes very high, mean scores, whereas students with African, central Asian and Pacific language backgrounds had relatively low mean scores. Language background differences tended to be larger for numeracy than for reading. Parental occupation and education accounted for some of the effects of language background for some groups, but not for Asian groups. Generally, language background effects cannot be attributed to the unequal distribution of language background groups across schools. When considering students' prior achievement, most language background effects, including all language backgrounds with strong negative effects, were reduced substantially often to statistical insignificance. The exceptions are Chinese and other East Asian and Southern Asian language backgrounds.
\end{abstract}

\section{Introduction}

Language background is a commonly used concept in both policy and research on student performance in Australia. The most common measure is Language Background Other than English (LBOTE) defined as students who speak a language other than English at home (ACARA, 2019a). LBOTE data is also used officially for school funding arrangements. Students with "low English proficiency" (defined as LBOTE students with at least one parent having a school education at or below Year 9 or equivalent) attract additional funding from the Australian Government (Department of Education, 2017). In the National Assessments—Literacy and Numeracy (NAPLAN), compared with non-LBOTE students, LBOTE students tend to show higher average scores than in numeracy, lower scores for reading, but there is some variation in this pattern across Year levels, achievement domains and educational jurisdictions (Chesters \& Daly, 2015; Creagh, 2014; Marks, 2014). 
Generally, across Year levels, achievement domains and jurisdictions, differences in performance between heterogenous LBOTE and non-LBOTE groups are consistently modest.

The LBOTE category is very heterogenous, comprising a wide variety of groups with quite disparate academic performances. The media tends to focus on the strong or weak performance of particular ethnic groups; using colourful phrases such as "tiger mums". 'Reference omitted to preserve the peer review process' (2010) found considerable variation in the effects of ethnicity (measured by father's country of birth) on Year 9 students' numeracy scores. Students from Malaysia and Hong Kong exhibited substantially higher (average) scores than students with Australian born fathers. Students with Lebanese, South and Central American, and Pacific backgrounds exhibited significantly lower mean numeracy scores. There were weaker ethnic differences in reading literacy than for numeracy. Creagh (2014) argued that the LBOTE category is too problematic for policy initiatives and demonstrated that the LBOTE category includes some of the most disadvantaged, notably refugee and, to a lesser extent, English as a Second Language (ESL) students. However, the number of refugee students is very small $(\approx 0.3$ per cent in her Queensland data) and ESL students may be exempted from NAPLAN if they had lived in Australia for less than 12 months or by agreement between the school and the student's parent or carer (ACARA, 2019b). ESL students may or may not be fluent in English.

There are three general explanations for immigrant differences in student achievement: socioeconomic background, schools and culture.

Immigrants to most Western countries tend to be less educated and have lower status jobs than native populations (Heath \& Brinbaum, 2014). Compared to non-immigrant students, immigrant students tend to have higher proportions of low socioeconomic background students (OECD, 2019, p. 183). For Australia, this would be generally true of students with African, Middle Eastern and Pacific language backgrounds. Socioeconomic explanations may also work in the other direction because of Australia's points based immigration scheme; the stronger performance of students from East and South East Asia may be because their parents are professionals or have sizable assets (Watkins, Ho, \& Butler, 2017). For the US, Lee and Zhou (2017) dismiss the role of cultural factors in "Asian academic excellence" and point to the immigration policy of allowing entry of highly educated Asian immigrants. Analysing data from the OECD's Programme for International Student Assessment (PISA), Levels, Dronkers and Kraaykamp (2008) conclude that socioeconomic differences between immigrant non-immigrant students contribute to immigrant students' scholastic achievement. 
However for Australia, Cobb-Clarke and Nguyen (2012) conclude that most of the gap between immigrant and non-immigrant youth in educational attainment could not explained by the higher levels of education of immigrant students' parents.

An alternative or complementary explanation is that poor performing immigrant students predominately attend low performing schools. Similarly, high performing ethnic groups may disproportionally attend well-resourced, high performing schools. Kao and Thompson (2003, p. 421) noted that researchers sometimes attribute the racial and ethnic differences that remain after controlling for socioeconomic background to differences in school quality. Cross-nationally, the weaker performance of immigrant students in PISA cannot be accounted for by their general overrepresentation in low performing schools (Levels et al., 2008; Marks, 2005). For the UK, Strand (2010) found no evidence that the performance gap between Black Caribbean and native students results from the minority group attending lower performing schools. For Australia, 'Reference omitted to preserve the peer review process' (2014, p. 237) found that, on average, LBOTE students attend higher performing schools which may account for some of the differences in performance between LBOTE and non-LBOTE students.

One of the major conclusions of the PISA study was the importance of immigrant students' country of origin rather than their country of destination (Carabaña, 2011; Dronkers, Levels, \& de Heus, 2014). This finding points to culture as an important factor. Ogbu (1978) distinguished between "voluntary" and "involuntary" minorities. Voluntary minorities assimilate into the host society and their children perform well in the educational system. Involuntary minorities exhibit attitudes and behaviours in opposition to the dominant culture, so their children tend to struggle in the education system (for a review of this approach see Warikoo \& Carter, 2009). However, cultural differences in student performance in Australia are more likely to simply reflect cultural differences in values, attitudes, and aspirations in education, rather than oppositional and assimilative cultures. A good example are the positive attitudes and values to education of Chinese students and their families (Chen \& Uttal, 1988). The cultural traits of cultural groups show a remarkable persistence across time and geographic location (Sowell, 1996).

This article contributes to our understanding of ethnic differences in achievement in Australia. First, it disaggregates the LBOTE category to twelve language background groups revealing great variation in NAPLAN performance by ethnicity. To our knowledge no study of students' NAPLAN performance has disaggregated the LBOTE category into smaller language or ethnic groups. Second, 
it examines the extent that language background differences in NAPLAN performance are accounted by socioeconomic factors, specifically measures of father's and mother's occupation and education. Third, it assesses the extent to which language differences in NAPLAN performance can be attributed to the uneven distribution of language background groups across schools. Do differential enrolments by ethnicity across schools account for, or at least contribute to, language background differences? Finally, it examines the origins of language background differences in achievement; the cultural processes involved may be contemporary or have occurred during early childhood. Although these analyses cannot conclude on which specific cultural factors are important to students' performance in NAPLAN, it can indicate if cultural factors influence student achievement at much the same time as testing, or at least two years before.

\section{Data, Measures and Methods}

\section{DATA}

The data comprise individual students attending Catholic schools in Victoria in 2017 and 2018. The data combine students' NAPLAN scores in numeracy and reading with administrative data. NAPLAN has been criticized for only measuring only limited aspects of what schools do, its inadequacy as a diagnostic tool, supposed adverse impacts on students and schools, and large errors in the estimates of student and school differences (Brady, 2013; Harris et al., 2013; Johnston, 2017; Wu, 2014). However, NAPLAN is the only instrument allowing parents, teachers and schools to objectively compare their students' performance against national and state-wide benchmarks. Furthermore, NAPLAN scores exhibit high reliability and are strong predictors of performance in the Year 12 university entrance performance (ACARA, 2018; Houng \& Justman, 2014). They also exhibit high stability evidenced by the strong correlations between prior achievement and achievement (Table 1).

The data analysed for this paper was obtained from the Catholic Education Commission of Victoria for the years 2017 and 2018. The total number of students with valid NAPLAN data in each Year level is around 15,000, around 30,000 for the two calendar years. Extensive preliminary analyses confirmed that the effects of language background and other covariates on NAPLAN scores are very similar for the two years. To our knowledge, NAPLAN data from other jurisdictions are not associated with the detailed ethnicity information available in the Victorian Catholic school databases. Obviously, the data from one school sector in one state is a limitation of this study, but it is unlikely 
that general patterns of language background differences in NAPLAN would be substantially different in other jurisdictions or Australia-wide.

\section{MEASURES}

\section{Student Achievement}

The measures of achievement and prior achievement are from the NAPLAN tests. NAPLAN tests are conducted on almost all students across Australia in Years 3, 5, 7 and 9. All students in the same year level are assessed on the same test items in five assessment domains. Each NAPLAN scale describes the development of student achievement from Year 3 through to Year 9. Scores range from 0 to 1000 . Student scores in each domain across the four Year levels are standardized to have a mean of 500 and a standard deviation of 100. Students in higher Year levels will show higher mean scores. These analyses conducted for this study are restricted to numeracy and reading. Prior achievement scores were collected two Year levels earlier for example, the variable "Prior Numeracy Year 5" are students' Year 3 numeracy scores.

Summary statistics for the NAPLAN measures are presented in Table 1. In accordance with the scales' construction, mean scores rise with Year level. In contrast, the variation (indicated by the standard deviation) declines. Table 1 shows that same-domain prior achievement correlate at between 0.70 and 0.85 with student achievement, higher for numeracy than for reading and showing an increase with Year level for numeracy.

\section{Language Background}

The measure of language background of the students was constructed from enrolment records from questions about whether the student spoke a language other than English at home and, if yes, what was the main language?

The language was coded according to the Australian Bureau of Statistics's (ABS, 2016) standard classification of languages. This hierarchical coding schema classifies over 400 languages into 51 narrow (2-digit) groups and nine broad (1-digit) regional language groups. For these analyses, high frequency language groups (Arabic, African, Chinese, English and Mon-Khmer languages) were categorised according to their 2-digit ABS codes. Otherwise, language groups were categorised according to their respective 1-digit category codes (East Asian, Southern Asian, Southern European etc.). So, in these analyses, the 1-digit language categories exclude students assigned to the narrower 
2-digit codes. For example, "Southwest and Central Asian" language background excludes Arabic, 1-digit language category "Other" (renamed as "Pacific Austronesia", see below) excluded African languages, "East Asian" excludes Chinese languages, "Southeast Asian" excludes Mon-Khmer languages and, of course, "Northern European" excludes English. These exclusions are noted in the tables, but not in the text. In the analysis there is one further departure from the ABS standard classification. The 1-digit ABS "Other" category includes the 2-digit codes for "Pacific Austronesia", "Oceanian Pidgins and Creoles", and "Papua New Guinea Languages" which were combined and with Australian Indigenous speakers (of which there were only a handful in each Year level). Since the group predominately comprises Samoan and Tongan speakers, (over 70 per cent) it is relabelled as "Pacific Austronesia". All students' language backgrounds were categorized into one of the 12 groups.

The language background categories and examples of languages within each group are presented in Table 2. The largest non-English language background groups are Southern European, Mon-Khmer, Southern Asian, Southwest and Central Asian, and Southeast Asian (Table 3).

\section{Background Variables}

The measure of Parents' Occupational status is based on enrolment records collected by schools. Parents are asked to categorise their occupation and whether they were not working, or the data was missing:

1. Senior management in large business organisation, government administration and defence, and qualified professionals

2. Other business managers, arts/media/sportspersons and associate professionals

3. Tradesmen/women, clerks and skilled office, sales and service staff

4. Machine operators, hospitality staff, assistants, labourers and related workers

8. Not in paid work in last 12 months

9. Not stated or unknown

The measures of parents' education for each parent were constructed from two variables, highest grade or Year level attained and post-school qualification resulted in the following scores for the 'years of education' ordinal measure': 
15. Bachelor's degree or above

12. Year 12 or equivalent

11. Year 11 or equivalent

10. Year 10 or equivalent

9. Year 9 or equivalent

The measure of parental education is the average number of years of education for both parents. If data from one parent is missing, then data from the other parent is used. If both parents' data are missing, then the variable was declared missing for that observation and the observation not included in the regression analyses. Table 1 includes summary statistics for the measure of parents' years of education.

Tables 1 to 3 about Here

\section{Analyses}

The analyses comprise successive regression models. The first model (results presented in Table 4) comprises only the language background variable and calendar year to index differences between the two cohorts. The estimates for each language background category are the differences in students' mean scores compared to the mean for students with an English-speaking language background. The intercept is the mean achievement scores for students from English-speaking background in $2007 .{ }^{2}$ Note that the estimates are equivalent to the category means for the two years combined. ${ }^{3}$ In the second model (Table 5), the measures of father's and mother's occupation and parents' education are added. Parents' occupations are entered as dummy variables with group 3 "Tradesmen/women, clerks and skilled office, sales and service staff" the reference category. Parents' education is a continuous variable centred at its mean, so that the intercept remains meaningful. In the second model, the intercept is the estimate for the average score of students from English speaking background with parents with occupations in the third occupational category with average levels of education. The fourth model (Table 6) is a multilevel model with random intercepts for schools. The specification of the fixed part of the model is the same as the specification for the second model. The final model (Table 7) also builds on the previous model with the addition of students' same-domain prior achievement score, centred. Note that it is not possible to include prior achievement for analysis 
of Year 3 NAPLAN performance. The coefficient for prior achievement is the expected change in NAPLAN score for a one unit increase in prior achievement score.

The results section refers to effect sizes to provide an indication of the magnitude of the effects. Cohens $d$ standardizes the difference between two means by dividing by the common standard deviation. An effect size of 0.2 is regarded as small, 0.5 medium and 0.8 large (Cohen, 1988). Standardized effects are referred to in the text for the continuous variables, parental education and prior achievement. ${ }^{4}$ The explanatory power of the models is indicated by the adjusted R square. For the models that include schools, the tables include the marginal and conditional (pseudo) R-squared values. The marginal $\mathrm{R}$ square is the variance explained by the fixed part of the model and the conditional $\mathrm{R}$ square is the variance accounted for by both the fixed and random components of the model (Nakagawa \& Schielzeth, 2013). The intraclass correlation (ICC) is the expected correlation in NAPLAN score for students randomly selected from the same school. The ICC is also the proportion of variance accounted for by schools of the total student-level variance in achievement, adjusted for the variables in the model (Snijders \& Bosker, 2012, p. 181).

\section{Results}

Table 4 presents the estimates for the effects of language background on students' NAPLAN scores. Students with a Chinese language background show the highest mean scores, 40 to 60 score points higher than that for English background students. These are large differences considering that the standard deviations for these measures range from 60 to just over 80 score points; the effect sizes are around 0.7 , often larger. The effects for a Chinese speaking background are considerably weaker for reading than for numeracy, but Chinese language background students comfortably outperform English background students in reading English. The effects of a Chinese language background show no clear pattern across Year levels, although its effects are weakest for Year 9. The other language background groups that exhibit substantially higher mean scores are the Eastern Asian language group and to a lesser extent the Mon-Khmer followed by Southern Asian language groups. The effects are consistently stronger for numeracy than reading and the numeracy effects are much weaker for Year 3 than for the three higher Year levels. A Northern European language background is associated with only marginally higher mean NAPLAN scores. In some instances, the effects are not statistically significant.

Tables 4 to 7 about Here 
Students with African, Pacific Austronesia and Southwest and Central Asian language backgrounds exhibit substantially lower mean scores than students from English speaking backgrounds. The effect sizes are large; over -0.80 for African, and Southwest and Central Asian (compared to English) language background students in numeracy, around -0.85 for Pacific and Austronesian language background students in Year 3 numeracy. The negative effects for Southwest and Central Asian language backgrounds are stronger for reading than for numeracy. The other language background groups with lower mean scores than English-speaking background students are Arabic, and to a lesser extent, Southern European language backgrounds. Students with Arabic speaking backgrounds fare worse in reading than in numeracy.

Table 5 presents the effects for language background net of parents' occupation and education. The parental occupational categories show small to moderate effects: larger for father's occupation compared to mother's occupation, and for father's occupation, larger at lower Year levels. For both Year 9 numeracy and reading the effects of father's occupation are weak. The largest effects are for no information on father's occupation (missing) which may partially reflect the lower scores of students from single-mother families. Parental education also has moderate effects on NAPLAN scores, the standardized effects of parental education on achievement are 0.22 for Year 3 numeracy and 0.23 for both Year 9 numeracy and reading. The addition of the socioeconomic background variables increased the variance explained from 2 to 6 per cent in the first model to between 9 and 14 per cent.

The strong positive effects on NAPLAN scores associated with a Chinese language background, at most, decline only marginally with the addition of socioeconomic background variables. Similarly, the addition of parents' occupation and education makes little difference to the sizable positive effects for Eastern Asian and Mon-Khmer language backgrounds. For these language backgrounds, there are several instances where the coefficients are larger when controlling for socioeconomic background. Net of parental occupation and education, the small positive effects of a Southeast Asian language background decline to statistical insignificance. For Southern Asian language backgrounds, the addition of the socioeconomic variables reduced the coefficients for numeracy by about one-quarter and the coefficients for reading more substantially; they became negligible.

The negative effects for African, Arabic, Pacific Austronesia, and Southwest and Central Asian language backgrounds decline by between one-third and one-half with the addition of parents' 
occupation and education. This indicates that a substantial part of the differences in NAPLAN scores between these language backgrounds and an English-speaking language background can be accounted for by socioeconomic background, but negative effects remain with effect sizes generally between 0.3 and 0.5. The smaller effects for a Southern European language background are largely unchanged with the addition of the socioeconomic variables.

Table 6 presents the estimates from the models that adjusts for socioeconomic background and schools. The addition of schools to the model has increased the (Conditional) R square values to between 10 and 17 per cent. As is the case in the previous analyses, there is a tendency for the model to explain less variation at higher Year levels. Schools (in the Victorian Catholic sector) account for little variation in NAPLAN achievement when controlling for socioeconomic background; the intraclass correlations range from 0.03 to 0.06 .

The addition of random effects for schools reduced the coefficients to varying extents depending on the language background group. For Chinese, Eastern Asian, Mon-Khmer and Southern Asian language backgrounds, the addition of random effects for schools, the language background effects decline only marginally, indicating that the strong performance of students with these backgrounds has little to do with the schools they attend. For some of these Asian language background groups, controlling for school differences increases the effects for language background indicating that students perform well, no matter which school they attend.

Similarly, the negative effects for Arabic and Pacific Austronesia language backgrounds are largely unaffected by including random effects for schools. Therefore, the distribution of students' language backgrounds across schools only marginally accounts for these students' lower average performance in NAPLAN tests. For an African language background, controlling for schools produces larger declines of between 10 and 20 per cent suggesting that schools contribute somewhat to their weaker performance in NAPLAN. For Southwest and Central Asian language backgrounds, controlling for school differences reduced the language background effects by about one-third suggesting there these students tend to be enrolled in lower performing schools. After controlling for socioeconomic background and schools, the negative effects of a Southwest and Central Asian language background are still sizable especially for reading.

Table 7 presents the estimates from the final model which adds same-domain prior achievement to the previous model. As expected, prior achievement has very strong effects on 
NAPLAN achievement scores with standardized effects ranging from 0.66 for Year 5 reading to 0.79 for Year 9 numeracy. Its addition dramatically increases the variance accounted for to between 60 and 70 per cent for numeracy (from 10 to 17 per cent in the previous model) and between 50 to 57 per cent for reading (from 10 to 13 per cent). The intraclass correlations were further reduced, less than 0.03 in five of the six analyses. The effects of parents' occupation and education are negligible and often statistically insignificant when controlling for prior achievement.

When controlling for same-domain prior achievement, there is little or no effect for language background for most language backgrounds. This is especially true of language backgrounds that in previous analyses showed strong negative effects on NAPLAN score. No language background has sizable negative effects on achievement, net of prior achievement. This is an important finding since it indicates that of the weaker performance of students with African, Arabic, Pacific Austronesia, and Southwest and Central Asia language backgrounds occur early in the school career, at or before Year 3. The weaker performances of these students in Years 5, 7 and 9 cannot be explained by schools' provision of teaching and learning in the same Year-level as that of NAPLAN testing or non-school factors.

In contrast, there are some language backgrounds which show non-trivial positive effects on NAPLAN performance, net of same-domain prior achievement. These language backgrounds are all Asian: Chinese, Eastern Asian, Mon-Khmer and Southern Asian. Such effects are smaller for reading than for numeracy and smaller for Year 9 than for Years 5 or 7. This indicates that students with these language backgrounds experience substantially stronger achievement growth than students from English-speaking and other language backgrounds. It is quite remarkable that students with these Asian language backgrounds exhibit stronger growth in reading, often from a higher base, than students with an English-speaking background.

\section{Discussion}

These analyses confirm the widely held view that the LBOTE category is too crude a measure for policy and research purposes because it aggregates language background groups that differ widely in performance. LBOTE should not be used to evaluate schools' resource needs, even if parental education is also considered. If language background is one of the criteria used for school funding, it needs to be disaggregated. Furthermore, comparisons between schools' NAPLAN results by 
education authorities, education stakeholders, commentators and parents (through the My Schools website) are likely to be misleading since students' language backgrounds are not considered.

The wide variation in NAPLAN performance by language background was anticipated, but not the magnitudes of the language background effects. The positive effects for several Asian language backgrounds are sometimes very large as are the negative effects for some other language backgrounds. Not all Asian groups exhibited high mean scores. A Southern Asian language background had more moderate effects on NAPLAN and the effects of a Southeast Asian (excluding Mon-Khmer) language background were small and often not significant. These patterns of performance across language groups is unlikely to be unique to Victorian Catholic schools.

The sheer size of language background effects means that they are unlikely to be accounted for by socioeconomic factors or schools. The strong performance of Chinese, Mon Khmer and East Asian language background students cannot be accounted for by their (on average) higher socioeconomic backgrounds. Therefore, the strong performance of Chinese, Mon Khmer and East Asian language background students is not because of Australia's immigration system which evaluates professional and highly educated applicants more favourably. For Southern Asian language backgrounds, socioeconomic background accounts for some of the effects. Socioeconomic background does account for about one-third to one-half of the negative effects of African, Arabic, Pacific and Austronesia, and Southwest and Central Asian language backgrounds. So, it appears that socioeconomic background cannot account for the language backgrounds that perform well in NAPLAN, but accounts somewhat for the weaker performance of some language background groups. However, large negative effects are found for some language backgrounds even when considering socioeconomic background.

Schools have little to do with the strong performance of students from Asian language backgrounds. They perform well in both low and higher performing schools. Similarly, the weaker performance of Arabic and Pacific Austronesia language backgrounds is not associated with schools. In contrast, the weaker performance of African and to a greater extent, Southwest and Central Asian, language background students appears to be more strongly associated with schools, since controlling for schools reduced the respective effects. Other data are required to confirm this finding before further investigating the relationships between schools and the performance of students with these language backgrounds. 
The finding that students with Asian, Eastern Asian, Mom-Khmer, and Southern Asian language backgrounds experience substantial achievement growth relative to English-speaking background students points to ongoing cultural influences that facilitate academic achievement. These influences may be high family and community expectations, students wishing to live up to these expectations, and community-wide positive attitudes about schooling and the value of education. Of course, it is very difficult to identify which cultural factors matter.

Often the weaker performance of some language background minorities is attributed to contemporary cultural factors: attitudes inimical to educational success, negative peer pressure on educational success, disruptive behaviour at school, and even gangs and criminal behaviour. These explanations pertain to adolescents and are not applicable to primary school students. However, the analyses presented in this paper demonstrate that the factors responsible for the lower academic achievement of several language background groups occur at Year 3 or earlier, well before adolescence, ruling out these and similar explanations. To understand the processes involved in the weaker performance of some language background, it would be useful to examine preschool and early childhood factors. Then we may have a better understanding of underachievement of some language background groups.

\section{References}

ABS. (2016). Australian standard classification of languages (ASCL). Cat No. 1267.0: Australian Bureau of Statistics.

ACARA. (2018). NAPLAN 2018 technical report. Sydney: Australian Curriculum, Assessment and Reporting Authority.

ACARA. (2019a). Glossary. Retrieved from https://www.myschool.edu.au/glossary/\#1

ACARA. (2019b). Student participation. Retrieved from https://www.nap.edu.au/naplan/schoolsupport/student-participation

Brady, L. (2013). NAPLAN: Critiquing the criticisms. Curriculum and Teaching, 28(1), 47-55. 
Carabaña, J. (2011). Why do the results of immigrant students depend so much on their country of origin and so little on their country of destination? In M. Pereyra, H.-G. Kotthoff, \& R. Cowen (Eds.), PISA under examination (Vol. 11, pp. 207-221): SensePublishers.

Chen, C., \& Uttal, D. H. (1988). Cultural values, parents' beliefs, and children's achievement in the united states and china. Human Development, 31(6), 351-358.

Chesters, J., \& Daly, A. (2015). The determinants of academic achievement among primary school students: A case study of the Australian capital territory. Australian Journal of Labour Economics, 18(1), 131-144.

Cobb-Clark, D. A., \& Nguyen, T. H. (2012). Educational attainment across generations: The role of immigration background. Economic Record, 88(283), 554-575.

Cohen, J. (1988). Statistical power analysis for the behavioral sciences (2nd ed.). New Jersey: Lawrence Erlbaum.

Creagh, S. (2014). A critical analysis of problems with the LBOTE category on the NAPLAN test. Australian Educational Researcher, 41(1), 1-23.

Department of Education. (2017). Schooling resource standard funding amount: 2015 update.

Canberra: Commonwealth Department of Education.

Dronkers, J., Levels, M., \& de Heus, M. (2014). Migrant pupils' scientific performance: The influence of educational system features of origin and destination countries. Large-scale Assessments in Education, 2, 3.

Harris, P., Chinnappan, M., Castleton, G., Carter, J., de Courcy, M., \& Barnett, J. (2013). Impact and consequence of Australia's National Assessment Program - Literacy and Numeracy (NAPLAN) - using research evidence to inform improvement. TESOL in Context, $23(1 / 2), 30-52$.

Heath, A. F., \& Brinbaum, Y. (Eds.). (2014). Unequal attainments: Ethnic educational inequalities in ten western countries. Oxford: Oxford University Press. 
Houng, B., \& Justman, M. (2014). NAPLAN scores as predictors of access to higher education in Victoria Melbourne Institute Working Paper Series (No. 22/14). Melbourne: Melbourne Institute of Applied Economic and Social Research, University of Melbourne.

Johnston, J. (2017). Australian NAPLAN testing: In what ways is this a 'wicked' problem? Improving Schools, 20(1), 18-34.

Kao, G., \& Thompson, J. S. (2003). Racial and ethnic stratification in educational achievement and attainment. Annual Review of Sociology, 29, 417-442.

Lee, J., \& Zhou, M. (2017). Why class matters less for Asian-American academic achievement. Journal of Ethnic and Migration Studies, 43(14), 2316-2330.

Levels, M., Dronkers, J., \& Kraaykamp, G. (2008). Immigrant children's educational achievement in western countries: Origin, destination, and community effects on mathematical performance. American Sociological Review, 73(October), 835-853.

Marks, G. N. (2005). Accounting for immigrant non-immigrant differences in reading and mathematics in twenty countries. Ethnic and Racial Studies, 28(5), 925-946.

Marks, G. N. (2010). Improvements over the educational career of immigrant students. Australian Journal of Education 54(2), 133-154.

Marks, G. N. (2014). Demographic and socioeconomic inequalities in student achievement over the school career. Australian Journal of Education, 58(3), 223-247.

Nakagawa, S., \& Schielzeth, H. (2013). A general and simple method for obtaining $\mathrm{R}^{2}$ from generalized linear mixed-effects models. Methods in Ecology and Evolution, 4(2), 133142.

OECD. (2019). PISA 2018 results: Where all students can succeed (Vol. II). Paris: OECD Publishing.

Ogbu, J. C. (1978). Minority education and caste: The American system in cross-cultural perspective. San Diego, CA: Academic Press.

Snijders, T., \& Bosker, R. J. (2012). Multilevel analysis: An introduction to basic and advanced multilevel modelling (Second ed.). Los Angeles: Sage. 
Sowell, T. (1996). Migrations and cultures: A world view. New York: Basic Books.

Strand, S. (2010). Do some schools narrow the gap? Differential school effectiveness by ethnicity, gender, poverty, and prior achievement. School Effectiveness and School Improvement,

21(3), 289-314.

Warikoo, N., \& Carter, P. (2009). Cultural explanations for racial and ethnic stratification in academic achievement: A call for a new and improved theory. Review of Educational Research, $79(1), 366-394$.

Watkins, M., Ho, C., \& Butler, R. (2017). Asian migration and education cultures in the anglo-sphere. Journal of Ethnic and Migration Studies, 43(14), 2283-2299.

$\mathrm{Wu}, \mathrm{M}$. (2014). Inadequacies of NAPLAN results for measuring school performance. Australia: Australian Senate.

Table 1: Summary Statistics for NAPLAN Domain and Year

\begin{tabular}{l|cccccc} 
Variable & N & Mean & St. Dev. & Min & Max & Correlation \\
\hline Numeracy Year 3 & 30,371 & 414.7 & 70.2 & 92.4 & 772.4 & - \\
Prior Numeracy Year 5 & 28,962 & 407.3 & 74.2 & 64.7 & 783.1 & \} 0.76 \\
Numeracy Year 5 & 30,891 & 499.0 & 62.1 & 195.5 & 777.8 & \\
Prior Numeracy Year 7 & 30,798 & 502.7 & 64.9 & 169.8 & 790.7 & \} 0.80 \\
Numeracy Year 7 & 32,580 & 556.3 & 62.9 & 216.9 & 894.8 & \\
Prior Numeracy Year 9 & 29,602 & 552.9 & 60.2 & 219.2 & 839.1 & \} 0.83 \\
Numeracy Year 9 & 30,898 & 595.7 & 57.3 & 311.1 & 882.8 & \\
Reading Year 3 & 30,416 & 443.7 & 84.2 & 0.0 & 849.1 & \\
Prior Reading Year 5 & 29,162 & 439.2 & 81.5 & 21.1 & 810.7 & \multirow{3}{*}{0.71} \\
Reading Year 5 & 31,014 & 515.7 & 75.2 & 0.0 & 907.0 & \\
Prior Reading Year 7 & 30,991 & 512.2 & 74.2 & 76.7 & 890.4 & \multirow{3}{*}{0.75} \\
Reading Year 7 & 32,729 & 552.4 & 63.7 & 11.8 & 836.2 &
\end{tabular}




\begin{tabular}{l|cccccc} 
Prior Reading Year 9 & 29,817 & 552.3 & 63.0 & 113.2 & 890.2 & \}$_{0.73}$ \\
Reading Year 9 & 31,062 & 588.7 & 61.2 & 102.5 & 849.0 & \\
Parents' Years of Education & 128,157 & 12.6 & 1.6 & 9.0 & 15.0 &
\end{tabular}

Note: Prior Achievement Data refers to Previous NAPLAN test

Table 2: Language Background Groups with Example Languages

\begin{tabular}{l|l|}
\multicolumn{1}{|l}{ Language Group or Language Region } & Example Languages \\
\hline African & $\begin{array}{l}\text { Somali, Swahili, Zulu } \\
\text { Arabic }\end{array}$ \\
Chinese & $\begin{array}{l}\text { Cantonese, Mandarin, Hakka } \\
\text { Eastern Asian (excl. Chinese) }\end{array}$ \\
Eastern European & $\begin{array}{l}\text { Hungarian, Russian, Polish, Ukrainian } \\
\text { Vietnamese, Khmer, Lao } \\
\text { Mon-Khmer }\end{array}$ \\
Northern European (excl. English) & $\begin{array}{l}\text { French, German, Dutch, Swedish, Gaelic, Afrikaans } \\
\text { Aboriginal Languages, Samoan, Tongan, Fijian, Maori, }\end{array}$ \\
Pacific Austronesia & $\begin{array}{l}\text { Papua New Guinea Languages } \\
\text { Burmese, Thai, Indonesian, Malay, Filipino }\end{array}$ \\
Southeast Asian (excl. Mon-Khmer) & Hindi, Tamil, Punjabi, Urdu, Sinhalese, Kashmiri \\
Southern Asian & $\begin{array}{l}\text { Italian, Greek, Spanish, Maltese } \\
\text { Surkish, Kurdish, Persian, Arabic, Armenian }\end{array}$ \\
Southern European &
\end{tabular}


Table 3: Frequencies for Language Background by NAPLAN Domain and Year

\begin{tabular}{|c|c|c|c|c|c|c|c|c|c|c|c|c|c|c|c|c|}
\hline \multirow{3}{*}{ Language Group or Language Region } & \multicolumn{8}{|c|}{ Numeracy } & \multicolumn{8}{|c|}{ Reading } \\
\hline & \multicolumn{2}{|c|}{ Year 3} & \multicolumn{2}{|c|}{ Year 5} & \multicolumn{2}{|c|}{ Year 7} & \multicolumn{2}{|c|}{ Year 9} & \multicolumn{2}{|c|}{ Year 3} & \multicolumn{2}{|c|}{ Year 5} & \multicolumn{2}{|c|}{ Year 7} & \multicolumn{2}{|c|}{ Year 9} \\
\hline & $\mathbf{N}$ & $\%$ & $\mathbf{n}$ & $\%$ & $\mathbf{N}$ & $\%$ & $\mathbf{N}$ & $\%$ & $\mathbf{N}$ & $\%$ & n & $\%$ & $\mathbf{n}$ & $\%$ & $\mathbf{n}$ & $\%$ \\
\hline African & 673 & 2.2 & 577 & 1.9 & 538 & 1.7 & 455 & 1.5 & 676 & 2.2 & 578 & 1.9 & 543 & 1.7 & 461 & 1.5 \\
\hline Arabic & 571 & 1.9 & 622 & 2 & 474 & 1.5 & 478 & 1.6 & 579 & 1.9 & 624 & 2 & 479 & 1.5 & 482 & 1.6 \\
\hline Chinese & 513 & 1.7 & 498 & 1.6 & 404 & 1.2 & 382 & 1.2 & 510 & 1.7 & 498 & 1.6 & 407 & 1.2 & 381 & 1.2 \\
\hline Eastern Asian (excl. Chinese) & 98 & 0.3 & 67 & 0.2 & 78 & 0.2 & 76 & $0.2 \vdots$ & 96 & 0.3 & 67 & 0.2 & 78 & 0.2 & 76 & 0.2 \\
\hline Eastern European Language & 588 & 1.9 & 603 & 2 & 500 & 1.5 & 557 & $1.8 \vdots$ & 588 & 1.9 & 608 & 2.0 & 506 & 1.6 & 560 & 1.8 \\
\hline English & 21703 & 71.5 & 22169 & 71.8 & 24568 & 75.4 & 23240 & $75.2 \vdots$ & 21723 & 71.4 & 22279 & 71.8 & 24683 & 75.4 & 23361 & 75.2 \\
\hline Mon-Khmer & 1160 & 3.8 & 1349 & 4.4 & 1007 & 3.1 & 1114 & 3.6 & 1159 & 3.8 & 1343 & 4.3 & 1003 & 3.1 & 1121 & 3.6 \\
\hline Northern European (excl. English) & 147 & 0.5 & 154 & 0.5 & 151 & 0.5 & 131 & $0.4 \vdots$ & 147 & 0.5 & 155 & 0.5 & 152 & 0.5 & 127 & 0.4 \\
\hline Pacific Austronesia & 168 & 0.5 & 167 & 0.5 & 162 & 0.5 & 121 & $0.4 \vdots$ & 171 & 0.6 & 166 & 0.5 & 164 & 0.5 & 124 & 0.4 \\
\hline Southeast Asian (excl. Mon-Khmer) & 911 & 3 & 830 & 2.7 & 779 & 2.4 & 741 & $2.4 \vdots$ & 910 & 3 & 831 & 2.7 & 779 & 2.4 & 742 & 2.4 \\
\hline Southern Asian & 1419 & 4.7 & 1180 & 3.8 & 1232 & 3.8 & 818 & $2.6 \vdots$ & 1425 & 4.7 & 1181 & 3.8 & 1229 & 3.8 & 827 & 2.7 \\
\hline Southern European & 1942 & 6.4 & 2164 & 7 & 2337 & 7.2 & 2487 & $8.1:$ & 1955 & 6.4 & 2168 & 7.0 & 2352 & 7.2 & 2503 & 8.1 \\
\hline Southwest,Central Asian (excl. Arabic) & 478 & 1.6 & 511 & 1.7 & 350 & 1.1 & 298 & $1:$ & 477 & 1.6 & 516 & 1.7 & 354 & 1.1 & 297 & 1.0 \\
\hline Totals & 30371 & & 30891 & & 32580 & & 30898 & & 30416 & & 31014 & & 32729 & & 31062 & \\
\hline
\end{tabular}

Table 4: Language Background Differences in NAPLAN Performance

This article is protected by copyright. All rights reserved 


\begin{tabular}{|c|c|c|c|c|c|c|c|c|}
\hline 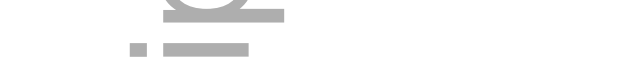 & \multicolumn{4}{|c|}{ Numeracy } & \multicolumn{4}{|c|}{ Reading } \\
\hline 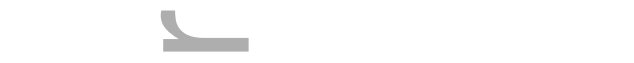 & Year 3 & Year 5 & Year 7 & Year 9 & Year 3 & Year 5 & Year 7 & Year 9 \\
\hline Constant & $419.3^{* * *}$ & $498.5^{* * *}$ & $558.0^{* * * *}$ & $593.6^{* * * *}$ & $447.8^{* * * *}$ & $516.8^{* * *}$ & $556.0^{* * * *}$ & $588.2^{* * *}$ \\
\hline Year 2018 (vs 2017) & $-4.8^{* * * *}$ & 0.7 & $-3.5^{* * *}$ & $5.3^{* * *}$ & -1.3 & $3.6^{* * *}$ & $-3.4^{* * *}$ & $4.8^{* * *}$ \\
\hline African & $-58.5^{* * *}$ & $-50.8^{* * *}$ & $-53.7^{* * * *}$ & $-47.5^{* * * *}$ & $-53.0^{* * * *}$ & $-61.2^{* * *}$ & $-53.1^{* * *}$ & $-42.7^{* * *}$ \\
\hline Eastern Asian (excl. Chinese) & $29.8^{* * *}$ & $60.9^{* * *}$ & $41.6^{* * *}$ & $45.1^{* * *}$ & 4.6 & $32.3^{* * *}$ & $13.7^{*}$ & $21.8^{* * *}$ \\
\hline Eastern European & -4.7 & $-5.2^{* *}$ & $-5.3^{*}$ & -2.0 & -3.5 & $-11.0^{* * * *}$ & $-6.1^{* *}$ & $-4.9^{*}$ \\
\hline Mon-Khmer & $9.2^{* * *}$ & $25.5^{* * *}$ & $33.8^{* * *}$ & $27.8^{* * *}$ & $-6.5^{* * *}$ & $4.3^{* *}$ & $6.6^{* * *}$ & $4.2^{* *}$ \\
\hline Northern European (excl. English) & $10.3^{*}$ & $14.1^{* * *}$ & 7.4 & 7.2 & 1.0 & $12.2^{* *}$ & $9.3^{*}$ & $9.6^{*}$ \\
\hline Pacific Austronesia & $-59.7^{* * * *}$ & $-47.2^{* * *}$ & $-48.2^{* * *}$ & $-45.0^{* * * *}$ & $-53.0^{* * *}$ & $-47.7^{* * *}$ & $-45.9^{* * *}$ & $-34.7^{* * *}$ \\
\hline Southwest and Central Asian (excl. Arabic) & $-59.1^{* * *}$ & $-49.2^{* * *}$ & $-49.1^{* * *}$ & $-42.1^{* * *}$ & $-65.7^{* * *}$ & $-63.9^{* * *}$ & $-57.3^{* * *}$ & $-47.0^{* * *}$ \\
\hline Observations & 30,371 & 30,891 & 32,580 & 30,898 & 30,416 & 31,014 & 32,729 & 31,062 \\
\hline Adjusted $\mathrm{R}^{2}$ & 0.04 & 0.06 & 0.06 & 0.05 & 0.03 & 0.04 & 0.03 & 0.02 \\
\hline
\end{tabular}

Table 5: Language Background Differences in NAPLAN Performance, net of Parents' Occupation and Education

Numeracy
Reading

This article is protected by copyright. All rights reserved 


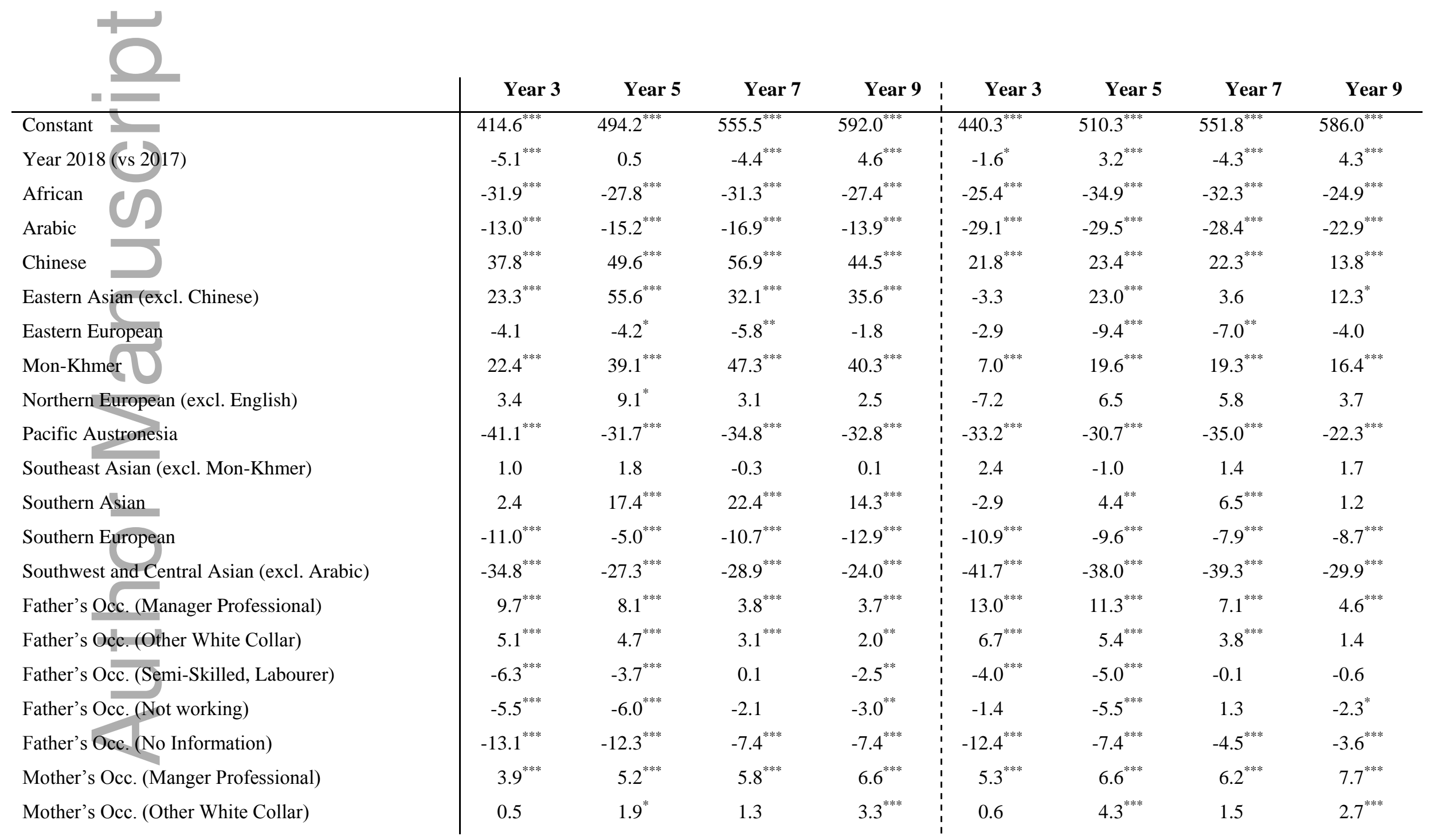

This article is protected by copyright. All rights reserved 
Mother's Occ. (Semi-Skilled, Labourer)

Mother's Occ. (Not working)

Mother's Occ. (No Information)

Average Parents' Years of Educ. (Centred)

Observations

Adjusted $\mathrm{R}^{2}$

Note: ${ }^{*} \mathrm{p}<0.1 ;{ }^{* *} \mathrm{p}<0.05 ;{ }^{* * *} \mathrm{p}<0.01$

\begin{tabular}{|cccc:cccc}
-1.6 & -1.8 & $-4.1^{* * *}$ & -1.2 & -2.0 & 0.5 & -1.4 & $-2.2^{*}$ \\
$-2.8^{* *}$ & 0.1 & $-6.8^{* * *}$ & $-2.8^{* *}$ & -0.9 & 0.5 & $-4.6^{* * *}$ & -2.2 \\
2.3 & 4.4 & -2.9 & 0.8 & $9.5^{* *}$ & 5.9 & 0.6 & -1.3 \\
$10.3^{* * *}$ & $9.0^{* * *}$ & $8.7^{* * *}$ & $8.3^{* * *}$ & $11.5^{* * *}$ & $10.5^{* * *}$ & $9.0^{* * *}$ & $8.3^{* * *}$ \\
30,155 & 30,423 & 30,968 & 30,032 & 30,195 & 30,544 & 31,112 & 30,199 \\
0.13 & 0.14 & 0.12 & 0.12 & 0.10 & 0.12 & 0.11 & 0.09
\end{tabular}

Table 6: Language Background Differences in NAPLAN Performance, net of Parents' Occupation and Education, and Schools

\begin{tabular}{|c|c|c|c|c|c|c|c|c|}
\hline \multirow{2}{*}{ 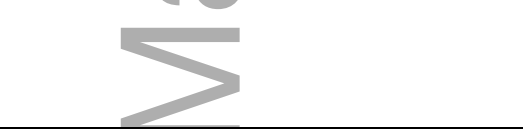 } & \multicolumn{4}{|c|}{ Numeracy } & \multicolumn{4}{|c|}{ Reading } \\
\hline & Year 3 & Year 5 & Year 7 & Year 9 & Year 3 & Year 5 & Year 7 & Year 9 \\
\hline Constant & $414.6^{* * *}$ & $493.6^{* * *}$ & $557.4^{* * * *}$ & $592.9^{* * * *}$ & $440.1^{* * * *}$ & $510.3^{* * *}$ & $553.3^{* * *}$ & $586.8^{* * *}$ \\
\hline Year 2018 (vs 2017) & $-4.8^{* * *}$ & 0.3 & $-4.1^{* * *}$ & $4.9^{* * *}$ & -1.4 & $3.1^{* * *}$ & $-4.1^{* * *}$ & $4.3^{* * *}$ \\
\hline African & $-25.0^{* * *}$ & $-23.4^{* * * *}$ & $-27.9^{* * *}$ & $-23.9^{* * *}$ & $-20.2^{* * *}$ & $-31.4^{* * *}$ & $-29.5^{* * *}$ & $-23.7^{* * *}$ \\
\hline Arabic & $-14.3^{* * *}$ & $-16.4^{* * *}$ & $-17.5^{* * *}$ & $-12.5^{* * *}$ & $-28.5^{* * *}$ & $-27.3^{* * *}$ & $-28.0^{* * *}$ & $-21.5^{* * *}$ \\
\hline Chinese & $35.3^{* * *}$ & $47.6^{* * *}$ & $54.0^{* * *}$ & $39.5^{* * *}$ & $18.6^{* * *}$ & $22.3^{* * *}$ & $18.7^{* * *}$ & $8.6^{* * *}$ \\
\hline Eastern Asian (excl. Chinese) & $23.3^{* * *}$ & $51.2^{* * *}$ & $30.7^{* * *}$ & $30.4^{* * *}$ & -4.3 & $20.5^{* *}$ & 2.0 & 7.0 \\
\hline Eastern European & -3.0 & -3.4 & -4.3 & -0.6 & -3.3 & $-8.7^{* * *}$ & $-5.2^{*}$ & -4.0 \\
\hline Mon-Khmer & $27.0^{* * *}$ & $38.2^{* * *}$ & $45.1^{* * *}$ & $37.1^{* * *}$ & $9.7^{* * *}$ & $18.9^{* * *}$ & $15.1^{* * *}$ & $10.6^{* * *}$ \\
\hline Northern European (excl. English) & 2.7 & 5.9 & 1.0 & 2.2 & -5.8 & 4.4 & 4.2 & 1.6 \\
\hline Pacific Austronesia & $-36.4^{* * *}$ & $-27.1^{* * *}$ & $-30.2^{* * *}$ & $-26.9^{* * *}$ & $-31.7^{* * *}$ & $-28.3^{* * *}$ & $-30.6^{* * *}$ & $-19.3^{* * *}$ \\
\hline Southeast Asian (excl. Mon-Khmer) & $5.3^{* *}$ & $7.0^{* * * *}$ & $4.8^{* *}$ & $4.9^{* *}$ & $4.7^{*}$ & 2.4 & $5.4^{* *}$ & $4.0^{*}$ \\
\hline
\end{tabular}

This article is protected by copyright. All rights reserved 


\section{Southern Asian}

Southern European

Southwest and Central Asian (excl. Arabic)

Father's Occ. (Manager Professional)

Father's Oce. (Other White Collar)

Father's Occ. (Semi-Skilled, Labourer)

Father's Occ. (Not working)

Father's Occ. (No Information)

Mother's Occ. (Manger Professional)

Mother's Occ. (Other White Collar)

Mother's Occ. (Semi-Skilled, Labourer)

Mother's Occ. (Not working)

Mother's Occ. (No Information)

Average Parents' Years of Educ. (Centred)

Observations

Marginal R Square

Conditional R Square

Intraclass Correlation

Note: ${ }^{*} \mathrm{p}<0.1 ;{ }^{* *} \mathrm{p}<0.05 ;{ }^{* * *} \mathrm{p}<0.01$

\begin{tabular}{|cccc:cccc}
$8.1^{* * *}$ & $22.6^{* * *}$ & $24.3^{* * *}$ & $15.1^{* * *}$ & 0.9 & $8.7^{* * *}$ & $8.3^{* * *}$ & 0.6 \\
$-13.2^{* * *}$ & $-7.6^{* * *}$ & $-11.4^{* * *}$ & $-14.0^{* * *}$ & $-14.8^{* * *}$ & $-11.4^{* * *}$ & $-9.6^{* * *}$ & $-10.5^{* * *}$ \\
$-20.2^{* * *}$ & $-18.4^{* * *}$ & $-19.8^{* * *}$ & $-15.6^{* * *}$ & $-29.7^{* * *}$ & $-30.5^{* * *}$ & $-33.1^{* * *}$ & $-23.9^{* * *}$ \\
$6.7^{* * *}$ & $5.3^{* * *}$ & $2.7^{* * *}$ & $2.8^{* * *}$ & $10.2^{* * *}$ & $9.1^{* * *}$ & $6.3^{* * *}$ & $4.4^{* * *}$ \\
$3.8^{* * *}$ & $3.3^{* * *}$ & $1.8^{* *}$ & 0.9 & $5.9^{* * *}$ & $4.1^{* * *}$ & $3.0^{* * *}$ & 1.0 \\
$-5.4^{* * *}$ & $-3.1^{* * *}$ & -0.4 & $-2.7^{* *}$ & $-3.0^{* *}$ & $-4.7^{* * *}$ & 0.1 & -0.02 \\
$-5.5^{* * *}$ & $-5.7^{* * *}$ & -2.2 & $-2.7^{* *}$ & -1.9 & $-4.9^{* *}$ & 1.3 & -1.4 \\
$-12.5^{* * *}$ & $-12.0^{* * *}$ & $-8.1^{* * *}$ & $-7.8^{* * *}$ & $-11.3^{* * *}$ & $-7.0^{* * *}$ & $-5.3^{* * *}$ & $-3.5^{* * *}$ \\
$2.9^{* *}$ & $3.9^{* * *}$ & $2.9^{* * *}$ & $2.7^{* * *}$ & $4.8^{* * *}$ & $5.7^{* * *}$ & $4.1^{* * *}$ & $5.8^{* * *}$ \\
-0.5 & 1.1 & -0.3 & $1.5^{* *}$ & -0.3 & $3.6^{* * *}$ & 0.4 & $2.1^{* *}$ \\
-1.2 & -0.6 & $-3.4^{* * *}$ & -0.7 & -1.6 & 1.2 & -0.7 & -1.4 \\
$-2.1^{* *}$ & 1.0 & $-6.0^{* * *}$ & -1.8 & -0.3 & 0.7 & $-3.9^{* * *}$ & $-2.4^{*}$ \\
-0.9 & 1.0 & $-5.2^{* *}$ & -3.0 & 6.5 & 3.0 & -1.1 & -2.9 \\
$9.4^{* * *}$ & $8.1^{* * *}$ & $7.6^{* * *}$ & $6.8^{* * *}$ & $10.4^{* * *}$ & $9.9^{* * *}$ & $7.7^{* * *}$ & $7.1^{* * *}$ \\
30,155 & 30,423 & 30,968 & 30,032 & 30,195 & 30,544 & 31,112 & 30,199 \\
0.10 & 0.12 & 0.10 & 0.09 & 0.08 & 0.10 & 0.08 & 0.07 \\
0.16 & 0.17 & 0.13 & 0.14 & 0.12 & 0.13 & 0.11 & 0.10 \\
0.061 & 0.062 & 0.036 & 0.058 & 0.043 & 0.031 & 0.034 & 0.039 \\
& & & & & &
\end{tabular}

This article is protected by copyright. All rights reserved 
Table 7: Language Background Differences in NAPLAN Performance, net of Parents' Occupation and Education, Schools and Prior Achievement

\begin{tabular}{|c|c|c|c|c|c|c|}
\hline \multirow{2}{*}{$C$} & \multicolumn{3}{|c|}{ Numeracy } & \multicolumn{3}{|c|}{ Reading } \\
\hline & Year 5 & Year 7 & Year 9 & Year 5 & Year 7 & Year 9 \\
\hline Constant & $497.6^{* * *}$ & $559.2^{* * *}$ & $596.9^{* * *}$ & $513.3^{* * *}$ & $555.2^{* * *}$ & $584.5^{* * * *}$ \\
\hline African & $-4.1^{* *}$ & $-5.0^{* * *}$ & -2.4 & $-7.9^{* * *}$ & $-8.0^{* * *}$ & $-7.9^{* * *}$ \\
\hline Arabic & -1.3 & -0.8 & -1.9 & $-7.7^{* * *}$ & $-4.9^{* *}$ & -2.7 \\
\hline Chinese & $24.8^{* * *}$ & $22.4^{* * *}$ & $11.7^{* * *}$ & $14.5^{* * *}$ & $15.3^{* * *}$ & $9.2^{* * *}$ \\
\hline Eastern European & 0.3 & -0.7 & 0.8 & -2.6 & 1.4 & 1.9 \\
\hline Mon-Khmer & $23.2^{* * *}$ & $22.5^{* * *}$ & $10.4^{* * *}$ & $12.5^{* * *}$ & $12.5^{* * *}$ & $8.6^{* * *}$ \\
\hline Northern European (excl. English) & -1.3 & -3.3 & 2.5 & 3.4 & 2.3 & 5.4 \\
\hline Pacific Austronesia & -3.4 & -4.4 & $-6.2^{* * *}$ & $-13.3^{* * *}$ & $-7.3^{* *}$ & 1.2 \\
\hline Southeast Asian (excl. Mon-Khmer) & $7.8^{* * * *}$ & $4.9^{* * * *}$ & $4.3^{* * *}$ & 1.8 & $6.6^{* * *}$ & $5.6^{* * *}$ \\
\hline Father's Occ. (Manager Professional) & $1.3^{*}$ & 1.2 & -0.02 & $2.2^{* *}$ & $3.1^{* * *}$ & 0.04 \\
\hline Father's Occ. (Other White Collar) & 0.3 & 0.6 & -0.9 & 0.5 & $1.4^{* * *}$ & -0.9 \\
\hline Father's Occ. (Semi-Skilled, Labourer) & $-1.7^{* *}$ & 0.7 & $-1.1^{*}$ & $-2.7^{* * *}$ & -0.1 & $1.7^{* *}$ \\
\hline Father's Occ. (Not working) & $-3.1^{* * *}$ & -0.00 & -1.1 & -1.5 & $1.7^{*}$ & -0.2 \\
\hline
\end{tabular}

This article is protected by copyright. All rights reserved 
Father's Occ. (No Information)

Mother's Occ. (Manger Professional)

Mother's Occ. (Other White Collar)

Mother's Occ. (Semi-Skilled, Labourer)

Mother's Oce. (Not working)

Mother's Occ. (No Information)

Average Parents' Years of Educ. (Centred)

Same-domain Prior Achievement (Centred)

Observations

Marginal R Square

Conditional R Square

Intraclass Correlation

Note: ${ }^{*} \mathrm{p}<0.1 ;{ }^{* *} \mathrm{p}<0.05 ;{ }^{*}{ }^{* *} \mathrm{p}<0.01$

\begin{tabular}{|c|c|c|c|c|c|}
\hline$-5.6^{* * *}$ & $-2.1^{* *}$ & $-2.2^{* * *}$ & -1.8 & $-2.0^{* *}$ & -1.5 \\
\hline $1.3^{*}$ & $1.2^{*}$ & 0.7 & $2.9^{* * *}$ & $1.7^{* *}$ & $2.4^{* * *}$ \\
\hline 0.3 & $-1.5^{* *}$ & -0.1 & $2.1^{* *}$ & 0.2 & 1.0 \\
\hline 1.0 & $-1.5^{* *}$ & 0.8 & $2.4^{* *}$ & -0.05 & 0.3 \\
\hline $1.5^{* *}$ & $-2.0^{* *}$ & 0.7 & 0.8 & 0.6 & 1.1 \\
\hline $4.5^{*}$ & -2.2 & -0.01 & -1.1 & 1.1 & -1.0 \\
\hline $2.9^{* * *}$ & $2.1^{* * *}$ & $1.7^{* * *}$ & $3.7^{* * *}$ & $2.6^{* * *}$ & $2.3^{* * * *}$ \\
\hline $0.59^{* * *}$ & $0.73^{* * *}$ & $0.75^{* * *}$ & $0.61^{* * *}$ & $0.60^{* * * *}$ & $0.68^{* * *}$ \\
\hline 28,570 & 29,262 & 28,802 & 28,764 & 29,448 & 29,009 \\
\hline 0.58 & 0.64 & 0.69 & 0.51 & 0.57 & 0.54 \\
\hline 0.60 & 0.65 & 0.70 & 0.52 & 0.57 & 0.55 \\
\hline 0.046 & 0.017 & 0.029 & 0.021 & 0.011 & 0.016 \\
\hline
\end{tabular}

This article is protected by copyright. All rights reserved 
Notes

Technical qualifications were not included in the measure of parents' education. Its inclusion reduced the effects of parents' education on NAPLAN score.

2 The intercept term is the predicted value for cases that scored zero on each predictor variable.

Mean for Language Category = Intercept + Language Category Regression Estimate (Model 1 only)

$4 \quad$ Standardized coefficients $(\beta)$ are obtained by multiplying the metric coefficient $(b)$ by the ratio of the standard deviations of the $\mathrm{x}$ and $\mathrm{y}$ variables, $\beta=b\left(\frac{\sigma_{X}}{\sigma_{Y}}\right)$.

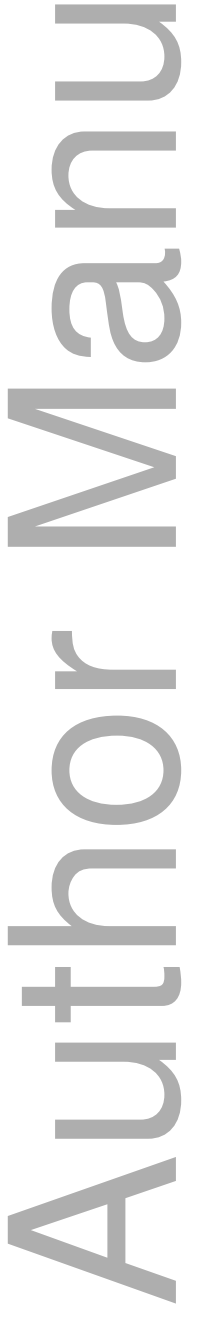




\section{University Library}

\section{- M M N E R VA A gateway to Melbourne's research publications}

Minerva Access is the Institutional Repository of The University of Melbourne

Author/s:

Marks, GN;Phillips, B

Title:

Accounting for language background differences in the Australian National Assessments Literacy and Numeracy

Date:

2020-03-02

\section{Citation:}

Marks, G. N. \& Phillips, B. (2020). Accounting for language background differences in the Australian National Assessments - Literacy and Numeracy. AUSTRALIAN JOURNAL OF SOCIAL ISSUES, 55 (3), pp.302-322. https://doi.org/10.1002/ajs4.108.

Persistent Link:

http://hdl.handle.net/11343/276852 